\title{
Vers un curriculum mondialisé? Une perspective comparative sur la recontextualisation des politiques curriculaires dans deux systèmes éducatifs francophones
}

\section{CHRONIQUE • Recherche étudiante}

Depuis une quinzaine d'années, les programmes de formation, les pratiques pédagogiques et l'évaluation des apprentissages font l'objet de réformes importantes dans de nombreux pays de l'OCDE. Les tensions se sont intensifiées depuis la fin des années 1990 quant à la question de ce qui « compte » comme savoir ou compétence légitime au XXI ${ }^{\mathrm{e}}$ siècle (Lingard et McGregor, 2014). Ces développements ont lieu dans un contexte où les comparaisons internationales (notamment en termes de performances des élèves de 15 ans) ont renforcé l'attention portée aux contenus d'enseignement et à leur transmission (Yates et Young, 2010). Ces pressions exogènes se sont ajoutées à des pressions endogènes liées à la massification de l'enseignement secondaire, à la diversification des publics scolaires et aux nouvelles fonctions de socialisation de l'école (Dubet, 2010), facteurs qui contribuent à faire du «curriculum » au sens large un enjeu majeur des politiques éducatives actuelles.

La convergence de tendances curriculaires mondiales conduit certains chercheurs à parler de la diffusion d'un « curriculum global » (Meyer, 2007), caractérisé par la centration sur les résultats d'apprentissage plutôt que les programmes disciplinaires, le développement de compétences transversales ou génériques et la promotion d'une pédagogie centrée sur l'apprenant. Or, ces réformes ne se déploient pas de façon uniforme et univoque. Des tendances contradictoires, des logiques en tension sous-tendent les changements contemporains, entre d'un côté le développement de compétences tournées vers la vie, l'appel à l'apprentissage actif, à la créativité, et de l'autre une centration 
sur les savoirs fondamentaux (notamment la maîtrise de la langue maternelle et les mathématiques) et sur les performances mesurées par des évaluations standardisées (Anderson-Levitt, 2003).

Malgré un intérêt renouvelé pour les questions dites « curriculaires », il existe peu de travaux empiriques abordant ces réformes curriculaires dans une perspective sociologique et aucune étude comparative systématique n'a été conduite en contexte francophone.

La méthodologie adoptée dans les analyses comparatives à "grande échelle » exclut toute possibilité d'analyse fine ou approfondie des curricula (Forquin, 2008). Or, une telle analyse pourrait faire apparaître la pluralité des formes que prennent ces changements dans différents espaces nationaux, ainsi que les processus d'hybridation entre différentes logiques et modèles, processus qui renforcent le caractère « composite» des politiques éducatives nationales (Maroy, 2006).

Cette recherche vise ainsi à interroger les transformations curriculaires contemporaines dans deux systèmes éducatifs francophones, la France et le Québec, où les programmes de l'enseignement secondaire ont subi au milieu des années 2000 une refonte majeure centrée sur le développement de compétences.

Deux questions guident notre recherche. D'abord, comment ces changements curriculaires se traduisent-ils dans le curriculum « formel » ou prescrit (Perrenoud, 1993) au niveau national, au-delà de l'usage de notions communes (par exemple, les compétences, la différenciation)? En second lieu, comment les acteurs aux prises avec ces prescriptions s'en saisissent-ils et leur donnent-ils sens, en particulier les conseillers pédagogiques et les inspecteurs pédagogiques régionaux chargés auprès des enseignants d'un travail de formation, d'encadrement et de diffusion des textes officiels?

Ces questions sont abordées en mobilisant principalement les apports de la sociologie du curriculum (Forquin, 2008), en particulier les repères théoriques de Bernstein (1990). Peu utilisés dans la recherche francophone portant sur les questions curriculaires, ils nous permettent d'analyser le «curriculum » dans ses trois dimensions : les contenus d'enseignement et leur organisation au sein du curriculum (le quoi), mais aussi les modalités de leur transmission (le comment) et leur évaluation. Ce cadre théorique nous fournit un langage de description commun pour analyser les textes curriculaires au-delà de leur contexte national. Il permet également de rendre compte des transformations qui affectent chaque dimension selon des temporalités et modalités différenciées et dans des sens parfois opposés.

Le concept de « recontextualisation» (Bernstein, 1990) est également central à cette recherche. Il renvoie d'une part à la recontextualisation d'idées en circulation internationale dans les politiques éducatives nationales (Ball, 1998), et d'autre part à la recontextualisation de ces politiques par les acteurs contribuant à assurer leur mise en œuvre, au niveau de la pratique pédagogique dans les établissements. Il s'agit alors de la circulation des « textes » qui constituent le curriculum formel, de leur diffusion, de leur réappropriation le long de la « chaîne curriculaire " (Forquin, 2008). Nous nous interrogeons sur le rôle joué par les "agents intermédiaires » dans la diffusion des prescriptions institutionnelles ainsi que des «bonnes pratiques » issues de la recherche, et dans l'accompagnement pédagogique auprès des enseignants.

Notre recherche s'appuie sur une méthodologie qualitative. Notre travail se fonde, d'une part, sur une analyse de contenu du curriculum prescrit ces dix dernières années au niveau du secondaire (premier cycle) en France et au Québec (documents législatifs et réglementaires mais aussi documents 
d'accompagnement), et d'autre part, sur un corpus d'entrevues semi-dirigées menées entre mars et septembre 2015 dans une académie et deux commissions scolaires auprès de conseillers et inspecteurs pédagogiques $(n=22)$, de formateurs $(n=5)$ et de cadres responsables des services éducatifs $(\mathrm{n}=10)$. L'analyse du corpus de textes et d'entrevues, en cours de réalisation, rend compte à la fois de dynamiques communes et de spécificités propres à chaque système, en vue de contribuer à une meilleure compréhension des politiques curriculaires contemporaines.

\section{Références}

Anderson-Levitt, K. M. (2003). A world culture of schooling. Dans K. M. Anderson-Levitt (dir.), Local meanings, global schooling: Anthropology and world culture theory (p. 1-26). New York, NY : Palgrave Macmillan.

Ball, S. J. (1998). Big policies/small world: An introduction to international perspectives in education policy. Comparative education, 34(2), 119-130. http://dx.doi.org/10.1080/03050069828225

Bernstein, B. (1990). The structuring of pedagogic discourse. New York, NY : Routledge.

Dubet, F. (2010). Déclin de l'institution et/ou néolibéralisme? Éducation et sociétés, 25(1), 17-34. http://dx.doi.org/10.3917/es.025.0017

Forquin, J.-C. (2008). Sociologie du curriculum. Rennes : Presses universitaires de Rennes.

Lingard, B. et McGregor, G. (2014). Two contrasting Australian Curriculum responses to globalisation: what students should learn or become. Curriculum Journal, 25(1), 90-110. http://dx.doi.org/10.1080/09585176.2013.872048

Maroy, C. (dir.). (2006). École, régulation et marché: une comparaison de six espaces scolaires locaux en Europe. Paris : Presses universitaires de France.

Meyer,J. W. (2007). World models, national curricula, and the centrality of the individual. Dans A. Benavot et C. Braslavsky (dir.), School Knowledge in Comparative and Historical Perspective (p. 259-271). Dordrecht : Springer. http://dx.doi.org/10.1007/978-1-4020-5736-6 16

Perrenoud, P. (1993). Curriculum : le formel, le réel, le caché. Dans J. Houssaye (dir.), La pédagogie : une encyclopédie pour aujourd'hui (2 ${ }^{\mathrm{e}}$ éd., p. 61-76). Paris : ESF.

Yates, L. et Young, M. (2010). Globalisation, knowledge and the curriculum. European Journal of Education, 45(1), 4-10. http://dx.doi.org/10.1111/j.1465-3435.2009.01412.x

\section{Pour citer cet article}

Mathou, C. (2016). Vers un curriculum mondialisé? Une perspective comparative sur la recontextualisation des politiques curriculaires dans deux systèmes éducatifs francophones. Formation et profession, 24(1), 74-76.

http://dx.doi.org/10.18162/fp.2016.a88 